\title{
PROPOSTA DE UM GERENCIAMENTO DE PROCESSOS DE REDUÇÃO DE CUSTOS NO ESTOQUE: UMA ANÁLISE BIBLIOMÉTRICA
}

\author{
Núbia Nunes \\ Universidade Federal do Rio Grande \\ Av. Itália, s/n - Km 8 - Carreiros, Rio Grande - RS \\ nubianuneseventos@gmail.com \\ André Andrade Longaray \\ Universidade Federal do Rio Grande \\ Av. Itália, s/n - Km 8 - Carreiros, Rio Grande - RS \\ andrelongaray@gmail.com \\ Paulo Roberto da Silva Munhoz \\ Universidade Federal do Rio Grande \\ Av. Itália, s/n - Km 8 - Carreiros, Rio Grande - RS \\ paulorsmunhoz@gmail.com \\ Déborah Lourenço Mendes \\ Universidade Federal do Rio Grande \\ Av. Itália, s/n - Km 8 - Carreiros, Rio Grande - RS \\ debloumendes@gmail.com \\ Kauê Costa Rego da Silva \\ Universidade Federal do Rio Grande \\ Av. Itália, s/n - Km 8 - Carreiros, Rio Grande - RS \\ kaue_costa@gmail.com
}

\section{RESUMO}

Diante do cenário atual no mercado competitivo das indústrias, as mesmas procuram minimizar seus custos, otimizando seus recursos. Um dos focos principais passa a ser a gestão dos estoques, com planejamento e controle priorizando reduzir seus desperdícios. O presente trabalho tem como objetivo analisar a produção cientifica no que se refere a gestão de controle do fluxo de materiais em empresas, investigar métodos que proporcionam redução dos custos e restrições de avarias. O estudo é realizado através da revisão bibliográfica dos artigos encontrados nas bases de dados do Portal de Periódicos da CAPES, Proquest, Science Direct, Scopus e Web of Science, o período da amostra foi livre, sem nenhuma restrição de área de pesquisa. O trabalho apresenta estudo exploratório, pesquisa qualitativa, mas predominantemente quantitativa, bases obtidas através de coleta de dados bibliográficos e posteriormente a sua análise.

Palavra-chave: Gestão de estoque; Avarias; Redução de custos; Bibliometria.

\section{ABSTRACT}


Given the current scenario in the competitive market of industries, they seek to minimize their costs, optimizing their resources. One of the main focuses becomes inventory management, with planning and control prioritizing reducing their waste. This paper aims to analyze the scientific production regarding the management of material flow control in companies, to investigate methods that provide cost reduction and breakdown restrictions. The study is conducted through the literature review of the articles found in the databases of the CAPES Journals Portal, Proquest, Science Direct, Scopus and Web of Science, the sample period was free, with no restriction of research area. The paper presents an exploratory study, qualitative but predominantly quantitative research, bases obtained through bibliographic data collection and later its analysis.

Keywords: Stock management; Damages; Cost reduction; Bibliometrics.

\section{Como Citar:}

SOBRENOME, Nome Autor 1; LONGARAY, André Andrade; MUNHOZ, Paulo Roberto da Silva; SILVA, Kauê Costa Rego da. Proposta de um gerenciamento de processos de redução de custos no estoque: uma análise bibliométrica. In: SIMPÓSIO DE PESQUISA OPERACIONAL E LOGÍSTICA DA MARINHA, 19., 2019, Rio de Janeiro, RJ. Anais [...]. Rio de Janeiro: Centro de Análises de Sistemas Navais, 2019.

\section{INTRODUÇÃO}

O ambiente corporativo, no transcorrer das últimas décadas mudou o cenário para um ambiente competitivo. As empresas buscam constantemente manter-se no mercado e passam a desenvolver estratégias de competitividade. Nesse sentido, para obter o mesmo, é necessário desempenhar e implementar vários processos, novas tecnologias e inteligência artificial. Contudo, de acordo com a Confederação Brasileira das Indústrias (CNI), atualmente especialistas estão requerendo profissionais mais criativos e que consigam desenvolver trabalhos em equipe [5].

Tendo em vista o cenário econômico, reduzir os custos foi um método adotado por boa parte dos empreendedores e gestores brasileiros durante os longos anos de crise que acometeram o país e que culminaram na pior recessão nacional desde 1930, após uma queda acumulada da atividade econômica de 7,2\% durante 2015 e 2016, segundo dados do IPEA (Instituto de Pesquisa Econômica Aplicada) e do IBGE (Instituto Brasileiro de Geografia e Estatística) [7].

Aumentar a produtividade é um dos desafios mais importantes para a indústria brasileira, em um momento de ajuste fiscal e retração econômica. Um projeto da Confederação Nacional da Indústria, em parceria com o Serviço Nacional de Aprendizagem Industrial (SENAI) e com o Instituto Euvaldo Lodi (IEL), mostrou que é possível mais que dobrar a produtividade e reduzir as despesas de produção em pouco tempo, com medidas simples e, mais importante, com baixo custo para as empresas [6].

Tendo em vista o aumento da produtividade e redução de custos, tem-se a aplicação de modelos de gestão como o Lean Thinking. O modelo de gestão empresarial Lean Thinking tem como objetivo aumentar a produtividade e a eficiência, e é baseado em dois pilares: melhoria contínua e respeito pelas pessoas. À vista disso, a ferramenta Lean thinking identifica sete desperdícios da produção, os quais são: defeitos, excesso de produção, espera, transporte, movimentação, processamento e estoque. Esta ferramenta busca transformar a realidade dos pequenos negócios, criando oportunidades para a melhoria da gestão, além de aumentar e fortalecer o comprometimento de seus profissionais [10]. 
Diante dessa conjuntura de controle de produção, tem-se a seguinte questão de pesquisa: quais os estudos, no cenário mundial, sobre a temática de modelos de gestão de estoques, com enfoque em minimizar os custos e avarias presentes no armazenamento e distribuição.

Frente ao exposto, o presente estudo tem como objetivo geral caracterizar a produção cientifica referente a temática de modelos de controle de custos e avarias em estoque. Os objetivos específicos são: identificar qual a relevância no cenário internacional, a partir da busca de publicações nas bases de dados: Scopus, Web of Science, Science Direct, Proquest e Portal de Periódicos CAPES, sobre modelos de controle de custos e avarias em estoque de modo a analisar a partir da análise bibliométrica quais os países, universidades, periódicos e autores mais relevantes dentro da temática. Ao realizar o mapeamento da produção cientifica internacional tem-se a finalidade de mensurar quais os modelos utilizados para realizar o controle de avarias em estoque estão sendo abordados em outros países.

O presente trabalho sendo dividido, primeiramente com referencial teórico acerca da importância do estudo. Seguidamente, apresenta-se a metodologia posta. Na continuidade é realizada a análise bibliográfica, apresentando a descrição da coleta de dados e resultados, neste último é proporcionada uma visão ampla e geral sobre as publicações selecionadas. Por fim, manifestam-se as considerações finais da pesquisa. $\mathrm{O}$ estudo abordado pormenorizado identifica os temas emergentes da análise da produção literária, nos quais podem servir de estruturas para futuras pesquisas sobre o tema.

\section{REFERENCIAL TEÓRICO}

O ambiente industrial está frequentemente em movimentação e transformações. Os variados processos realizados precisam ter uma sintonia estável, propondo gerenciamento de qualidade em todo processo produtivo, buscando otimização e vantagens competitivas. As empresas que conseguem melhorar seus produtos e serviços, bem como processos de produção, por meio de especialização, racionalização e tecnologia apresentam um grau maior de valor, ao comparar com seus concorrentes, e assim, alcançam vantagens competitivas [8].

Contudo a gestão de estoque é umas das atividades com maior relevância para obter resultados proveitosos. Desse modo, estoque pode ser definido como a acumulação de recursos materiais em um sistema de transformação, em alguns casos é utilizado para descrever qualquer recurso armazenado. Todavia, existem várias razões para que ocorra o desequilíbrio entre a taxa de fornecimento e a demanda do processo [12].

À vista disso, há quatro tipos de estoques: estoque isolador, estoque de ciclo, estoque de antecipação e estoque de canal. O estoque isolador tem como propósito compensar as incertezas inerentes a fornecimento e demanda. O estoque de ciclo é utilizado quando um ou mais estágios na produção não podem fornecer todos os itens que produzem simultaneamente. O estoque de antecipação é utilizado para compensar diferenças de ritmo de fornecimento e demanda. E por fim, o estoque no canal é utilizado quando o material não pode ser transportado instantaneamente entre o ponto de fornecimento e o ponto de demanda [12].

Para que se tenha uma gestão de estoque correta, é necessário a disponibilidade de informações para determinar o que manter em estoque, o que será abastecido e quando requisitar. Contudo, o maior problema dos gestores está principalmente em manter o equilíbrio entre o estoque e o consumo, desse modo, é necessário levar em conta o tipo de demanda e o controle de entradas e saídas no estoque. Uma vez que está gestão seja incorreta, a empresa sujeita-se a muitos prejuízos, como aumento de cancelamento de 
pedidos, paralisação da produção por falta de matéria-prima ou materiais semiacabados e a falta de espaço adequado gerando perda de itens devido ao armazenamento inadequado [3].

À vista disso, resume-se a atividade de gestão como uma ferramenta que visa o gerenciamento de estoques por meio de técnicas que permitam manter o equilíbrio com o consumo, definindo parâmetros e níveis e acompanhando sua evolução [13]. As principais funções no controle de estoque é determinar "o que" deve permanecer em estoque, em número de itens; "quando" se deve reabastecer os estoques em periodicidade; “quanto” de estoque será necessário para um período predeterminado; e a quantidade de compra. Acionar o Departamento de Compras para executar aquisição de estoque, receber, armazenar e atender os materiais estocados de acordo com as necessidades é também uma função do controle de estoque [1].

A gestão de estoque é item fundamental na manutenção da organização de um armazém, bem como na otimização da produtividade operacional. É por meio de um processo de estocagem que se pode ganhar proveito na ocupação do espaço, na utilização dos recursos operacionais, na otimização do tempo do pessoal e facilitar no processo de separação de pedidos [4].

No que tange a gestão correta do estoque, deve-se considerar também o processo de armazenagem. Desse modo, a armazenagem tem como proposito promover a disponibilidade de materiais para a indústria e para os clientes, contribuindo com a funcionalidade da cadeia de suprimentos. A cadeia de suprimento de uma organização, é comporta pela a logística de suprimentos e a logística de distribuição. A logística de suprimentos é responsável por gerenciar os materiais entre as fontes de suprimento e empresa. Bem como, a logística de distribuição tem por objetivo gerenciar as operações entre os pontos de processamento da empresa e seus clientes. À vista disso, a armazenagem está presente na logística de distribuição.

\section{METODOLOGIA}

$\mathrm{O}$ enquadramento metodológico da pesquisa é definido a partir do propósito do projeto, do caráter, do delineamento da pesquisa, das técnicas de coleta e da análise de dados a ser utilizado [9].

O propósito da pesquisa é obter informações sobre determinada população. $\mathrm{Na}$ presente pesquisa, a população é os artigos publicados sobre teoria das filas, cuja amostra é os artigos selecionados nas bases de dados Scielo, Google acadêmico e Web of Science [9].

Quanto ao caráter, o estudo é quantitativo, pois a pesquisa bibliométrica busca informações transformando em dados discretos para posteriormente realizar análise estatística. A bibliometria consiste na aplicação de técnicas estatísticas e matemáticas para descrever aspectos da literatura e de outros meios de comunicação [2].

A respeito do delineamento a pesquisa é considerada descritiva, porque busca contabilizar em que proporção ou frequência certos eventos acontecem. Com relação a técnica de coleta de dados, a pesquisa adequada é a bibliográfica, pois é através da busca em artigos que são coletadas as informações. No que tange à análise dos dados, foi predominantemente quantitativa, fazendo uso da técnica de bibliometria.

\section{ANÁLISE BIBLIOMÉTRICA}

A análise bibliométrica tem como objetivo realizar a quantificação da produção científica por meio da análise estatística. Neste sentido, na bibliometria ou análise bibliométrica, os dados quantitativos são contabilizados a partir das publicações ou elementos que reúnem uma série de técnicas estatísticas [11].

À vista disso, o presente estudo, será divido em duas etapas. A primeira etapa 
apresenta a coleta dos dados a partir das bases de dados: Portal de Periódicos CAPES, Proquest, Science Direct, Scopus e Web of Science. E por fim, a segunda etapa constitui na análise dos dados por meio da análise bibliométrica.

\subsection{Coleta de DAdOS}

Para realizar a coleta de dados foram utilizadas as bases de pesquisa: Portal de periódicos da CAPES, Proquest, Scopus, Science Direct e Web of Science. Realizou-se três etapas com combinações diferentes, adotando as palavras-chave: Breakdowns, Stock Control, Inventory Cost, Damage, Packaging, Bagging, Inventory Strategy, Inventory Management e Inventory Logistics.

No primeiro momento, aplicou-se o método utilizando três palavras e a combinação entre duas elas. $\mathrm{O}$ arranjo inicial se fez com a primeira palavra combinando com a segunda, a primeira com a terceira, a segunda com a terceira. Com o decorrer da pesquisa, notou-se que os resultados ficaram muito abrangentes de modo que poucos se alinhavam com a pesquisa, resultando 176.304 documentos.

Então, optou-se por mudar o método empregado: nessa segunda etapa utilizou-se as mesmas três palavras, porém alterou-se a combinação entre elas. Contudo, o resultado também não foi satisfatório: em duas das quatro bases de dados os resultados não foram consideráveis. A diferença de resultados provenientes da Web of Science e a Scopus, delas com as outras duas foi muito expressiva, levando os autores a crer que ainda haveria formas de aprimorar o estudo.

Observou-se que os resultados poderiam ficar mais ajustados, alterando uma das bases de dados e realizando a combinação apenas entre duas palavras-chave. Portanto, na terceira etapa, a combinação foi entre duas palavras-chave e houve substituição da base de dados Portal de periódicos da CAPES pela base de dados Proquest. Logo, a soma dos documentos sucedeu em 18.728. Subsequente as modificações, foram feitos os primeiros filtros, sendo postergados os blogs, patentes, livros, priorizando artigos e publicações em revistas. Com este processo seletivo resultando em 4.451 documentos;

Posteriormente a filtragem, os documentos duplicados foram descartados, assim, resultando 3.197 documentos. A partir da leitura dos títulos, reduziram para 256 documentos alinhados à pesquisa. Por fim, fez-se a leitura dos resumos, resultando em 21 artigos, sendo os demais excluídos devido não estarem em conformidade com o tema de pesquisa. Os artigos excluídos limitaram-se a temas como direcionamento ao cliente final, consumidores insatisfeitos, logística reversa, marketing de embalagens. A Tabela 1 apresenta os resultados da pesquisa realizada.

Tabela 1- Total de Artigos Pesquisados

\begin{tabular}{cc}
\hline Tipo de Filtragem & Número de Artigos \\
\hline Total duplicados & 3.197 \\
Total títulos & 256 \\
Total alinhados & 21 \\
\hline \multicolumn{2}{c}{ Fonte: Dados da Pesquisa }
\end{tabular}

Após o processo de filtragem, observou-se que a combinação das palavras-chave que mais ajustaram para alcançar a amostragem de 21 artigos foi packaging and inventory logistics que representa $43 \%$ da amostra total, as demais combinações Packaging and Inventory Cost e Packaging and Physical Stock representam 24\% da amostra, restando 9\% da combinação Packaging and Inventory Management.

A base que apresentou menor quantidade de amostras alinhadas a pesquisa foi a 
Scopus sem nenhum artigo e a que obteve a maior representatividade quantitativa do total selecionado foi a Web of Science com dez artigos, possuindo 48\% da amostra alinhada a pesquisa. Os artigos que compõem o portfólio bibliográfico estão dispostos no Apêndice A.

\section{RESULTADOS E DISCUSSÕES}

Na presente seção será feita a análise bibliométrica dos artigos presentes no portfólio. Dessa forma, será feito o estudo acerca da produção e autoria, conteúdo, e a citação dos artigos.

\subsection{ESTUdO DE PRODUÇÃO E AUTORIA}

A presente seção tem por objetivo apresentar a análise do número de publicações por ano, países que apresentaram maior representatividade nas publicações, universidades bem como, os autores que mais contribuíram com o tema de pesquisa.

Com propósito de avaliar a produção cientifica sobre as aplicações e métodos de gestão de estoque, com finalidade de reduzir desperdícios e avarias, construiu-se um histograma de acordo com o número de publicações especificada por ano. Na coleta de dados não se fez nenhuma restrição de tempo, os artigos alinhados, desse modo, os estudos presentes no portfólio bibliográfico contemplam os anos de 1960 a 2018. De acordo com a pesquisa, observou-se que a partir do ano de 2015 a tendência de crescimento de pesquisas relacionadas a diminuição de custos em gestão de estoque (Figura 1).

Figura 1- Frequência de publicações por ano

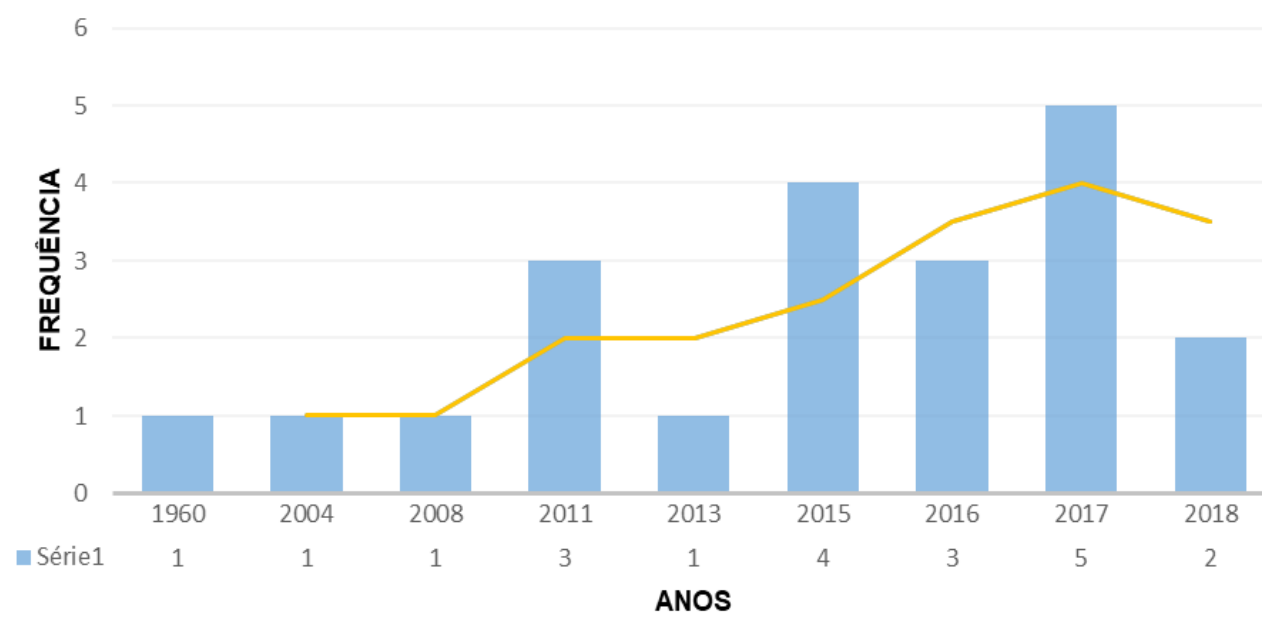

Fonte: Dados da Pesquisa

No que compete a quantificação dos países dos artigos analisados, identificou-se um total de 13 países. Os países que mais pesquisaram sobre a temática foram: China, Itália e Estados Unidos da América, onde a China atingiu maior representatividade com 23,80\%, com 5 artigos no portfólio, conforme demonstrado na Tabela 2.

Tabela 2 - Países com maior número de participações no portfolio

\begin{tabular}{ccc}
\hline Países & Número de Artigos & $\%$ \\
\hline China & 5 & $23,80 \%$ \\
Itália & 4 & $19,04 \%$
\end{tabular}




\begin{tabular}{ccc} 
Estados Unidos da América & 3 & $14,28 \%$ \\
Inglaterra & 3 & $14,28 \%$ \\
Hungria & 3 & $14,28 \%$ \\
\hline \multicolumn{2}{c}{ Fonte: Dados de pesquisa }
\end{tabular}

Ao proceder análise das instituições que mais produziram sobre o tema de pesquisa, foram levantadas 21 instituições atreladas aos primeiros autores de cada publicação. À vista disso, três universidades apresentaram maior representação onde a Universita' di Padova, School of Management e Fudan University contribuíram com 23,80\% do portfólio bibliográfica, com 5 artigos.

Ao realizar um estudo dos autores mais prolíferos, notou-se que o autor o Ishikawa, K. apresentou quatro artigos sobre a temática, com uma representatividade de 19,04\%. Após, o autor Andriolo, A, L contribuiu com três artigos e Brahimi, N., Cui, L. G e Olah, J., apresentaram dois artigos acerca do tema da pesquisa. Os demais autores do portfólio bibliográfico apresentam um artigo, desse modo, não estão representados na Tabela 3.

\begin{tabular}{ccc}
\multicolumn{3}{c}{ Tabela 3 - Número de publicações por autor } \\
\hline Autores & Participações & $\%$ \\
\hline Ishikawa, K & 4 & $19,04 \%$ \\
Andriolo, A. & 3 & $7,00 \%$ \\
Brahimi, N & 2 & $9,50 \%$ \\
Cui, L. G. & 2 & $9,50 \%$ \\
Olah, J. & 2 & $9,50 \%$ \\
\hline Fonte: Dados de pesquisa
\end{tabular}

\subsection{ANÁLISE DE CONTEÚDO}

A presente seção apresenta os dados de análise ao número de autores por artigo, bem como, as revistas de maior representatividade do portfólio bibliográfico.

Realizou-se a análise do número de autores por publicação, onde obteve-se sete artigos escritos por dois e três autores, contribuindo com 33,3\% do portfólio, respectivamente. Por seguinte, tem-se três artigos inscritos por quatro autores e dois artigos inscritos por um e cinco autores (Tabela 4).

\begin{tabular}{ccc}
\multicolumn{3}{c}{ Tabela 4 - Número de autores por publicações } \\
\hline Número de autores & Frequência & \% \\
\hline 2 & 7 & $33,30 \%$ \\
3 & 7 & $33,30 \%$ \\
4 & 3 & $14,30 \%$ \\
5 & 2 & $9,55 \%$ \\
1 & 2 & $9,55 \%$ \\
\hline \multicolumn{4}{r}{ Fonte: Dados de pesquisa }
\end{tabular}

A Tabela 5 demonstra as revistas que apresentaram maior número de publicações 
por revista. A revista que possuiu maior representatividade quantitativa dentro da amostra foi o International Journal of Production Economics, com cinco artigos.

Tabela 5 - Número de publicações por revistas

\begin{tabular}{ccc}
\hline Revistas & Frequência & $\%$ \\
\hline International Journal of Production Economics & 5 & $23,80 \%$ \\
International Journal of Production Research & 4 & $19,04 \%$ \\
International Journal of Industrial Ergonomics & 2 & $9,52 \%$ \\
International Journal of Quality \& Reliability Management & 2 & $9,52 \%$ \\
\hline
\end{tabular}

Fonte: Dados de pesquisa

Tabela 6 está apresentada 19 artigos e seu respectivo número de citações. Como parâmetro, utilizou o Google Acadêmico como ferramenta para análise do número de citações dos artigos do portfólio. O artigo mais citado foi Optimal policies for a multiechelon inventory problem com 2071 citações. Dois artigos não apresentaram nenhuma citação, os quais foram: Aggregate planning problem from sustainability perspective e Online inventory control in single-echelon systems with time-dependent environment over multiple periods.

Tabela 6 - Citações por artigo.

\begin{tabular}{|c|c|}
\hline Artigos & Citações \\
\hline Optimal policies for a multi-echelon inventory problem & 2071 \\
\hline To pull or not to pull: what is the question? & 506 \\
\hline Environmentally responsible inventory models: Non-classical models for a non-classical era & 214 \\
\hline $\begin{array}{l}\text { An inventory control system for spare parts at a refinery: An empirical comparison of different } \\
\text { re-order point methods }\end{array}$ & 181 \\
\hline Management accounting and control practices in a lean manufacturing environment & 120 \\
\hline Assessing the environmental impact of integrated inventory and warehouse management & 35 \\
\hline $\begin{array}{l}\text { Investigation of RFID investment in a single retailer two-supplier supply chain with random } \\
\text { demand to decrease inventory inaccuracy }\end{array}$ & 24 \\
\hline Multi-item production routing problem with backordering: a MILP approach & 23 \\
\hline A new bi-objective approach for including ergonomic principles into EOQ model & 16 \\
\hline Environmentally conscious supply chain management & 16 \\
\hline Optimal discounting and replenishment policies for perishable products & 13 \\
\hline . MIP models for production lot sizing problems with distribution costs and cargo arrangement & 9 \\
\hline Shelf life extending packaging, inventory control and grocery retailing & 9 \\
\hline Ergo-lot-sizing: Considering ergonomics in lot-sizing decisions & 7 \\
\hline Understanding young Chinese wine consumers through innovation diffusion theory & 6 \\
\hline $\begin{array}{l}\text { Diagnosis of operational malfunctions and failures by the FMEA method in the industrial } \\
\text { robots sector }\end{array}$ & 4 \\
\hline Inventory methods in order to minimize raw materials at the inventory level in the su & 4 \\
\hline
\end{tabular}




\section{SPOLM2O19}

XIX SIMPÓSIO DE PESQUISA OPERACIONAL E LOGISTICA DA MARINHA

The impact of cooperation mechanism on the chaotic behaviours in nonlinear supply chains

Fonte: Dados de pesquisa

\section{RESULTADOS E DISCUSSÕES}

Este estudo teve como objetivo a coleta de pesquisas científicas referentes ao controle de avarias de embalagens no estoque, afim de encontrar um modelo ótimo que diminuía os desperdícios. Para alcançar tal objetivo, foi realizada uma pesquisa bibliográfica de artigos disponíveis em bases de online.

Nesse sentido, o portfólio bibliográfico resultou em 21 artigos alinhados à pesquisa. Como exposto ao decorrer do estudo, fez-se a busca em cinco bases de dados, Portal de Periódicos CAPES, Proquest, Science Direct, Scopus e Web of Science., utilizando as combinações de palavras-chave.

Ao avaliar os resultados da análise bibliométrica, verificou-se que os estudos presentes no portfólio bibliográfico contemplam os anos de 1960 a 2018. De acordo com a pesquisa, observou-se que a partir do ano de 2015 a tendência de crescimento de pesquisas relacionadas a diminuição de custos em gestão de estoque.

Os países que mais pesquisaram sobre a temática foram: China, Itália e Estados Unidos da América, onde a China atingiu maior representatividade com 23,80\%, com 5 artigos no portfólio.

As universidades que obtiveram mais destaque na pesquisa em análise foram Universita' di Padova, School of Management e Fudan University contribuíram com 23,80\% do portfólio bibliográfica, com 5 artigos.

Os autores que apresentaram maiores números de artigos escritos e que estão incluídos no portfólio de pesquisa são Ishikawa, K. com quatro artigos, Andriolo, A. com três artigos e Brahimi, N., Cui, L. G e Olah, J. com dois artigos. Verificou-se que os artigos encontrados foram escritos por predominantemente por dois e três autores.

No que tange a análise das revistas, a revista que possuiu maior representatividade quantitativa dentro da amostra foi o International Journal of Production Economics, com cinco artigos.

A partir da ferramenta de citações do Google Acadêmico, obteve-se o artigo Optimal policies for a multi-echelon inventory problem foi o mais citado com 2071 citações.

Como limitação de pesquisa ressalta-se a dificuldade em obter publicações direcionadas sobre o assunto, foi possível identificar por meio da bibliometria, o gargalo na amostra: há uma quantidade restrita de artigos publicados com modelos que estudam sobre avarias de armazenamento e desperdícios com embalagens primárias. Como recomendação para pesquisas futuras, a amostra pode ser mais abrangente envolvendo maiores combinações de palavras-chave e incluir um maior número de bases de pesquisas para realizar a coleta de dados.

\section{REFERÊNCIAS BIBLIOGRÁFICAS}

[1] ARAÚJO, A. A. Gestão de estoques: controle e movimentação de materiais em um almoxarifado. Disponível em: <http://www.repositorio.uniceub.br/bitstream/235/11572/1/51500507.pdf>Acesso em: 21 de maio de 2018. 
[2] ARAÚJO, C. A. Bibliometria: evolução histórica e questões atuais. Em questão, v. 12, n. 1, p. 11-32, 2006.

[3] ALMEIDA, H. J.; SARAIVA, J. F.; SOUZA, M. S. de. Uma avaliação do processo de gestão e controle de estoques realizado por uma empresa prestadora de serviços logísicos in house. REFAS: Revista FATEC Zona Sul, v. 2, n. 1, p. 1-21, 2015.

[4] BANZATO, E.; JUNIOR, E.C; BANZATO, J.M.; MOURA, R.A. \& RAGO, S. F. T. Atualidades na Armazenagem. 1. ed. São Paulo: 2003.

[5] CNI - Confederação Nacional da Indústria. Momento de parar e pensar em produtividade é agora, na crise. Disponível em:

$<$ http://www.fiepi.com.br/fiepi/noticias/geral/entrevista-momento-de-parar-e-pensarem-produtividade-e-agora,-na-crise-3212.html> Acesso em: 20 de maio de 2018.

[6] CNI - Confederação Nacional da Indústria. Profissionais que dominam competências da indústria 4.0 vão ter mais chance no mercado Disponível em: $<$ https://noticias.portaldaindustria.com.br/entrevistas/rafael-lucchesi/profissionais-quedominarem-competencias-da-industria-40-vao-ter-mais-chances-no-mercado $>$ Acesso em: 25 de junho de 2018.

[7] IPEA. Instituto de Pesquisa Econômica Aplicada. Carta de Conjuntura. Disponível em :<http://www.ipea.gov.br/cartadeconjuntura/> Acesso em 22 de junho de 2018.

[8] OLIVEIRA, O. J. Gestão da Qualidade: Tópicos Avançados. Cengage Learning Editores, 2004.

[9] ROESCH, S. M. A. Projetos de estágio e de pesquisa em administração. São Paulo: Atlas, 2010.

[10] SEBRAE. Conheça a filosofia Lean e diminua os gastos da sua empresa.

Disponível em: <www.sebrae.com.br/sites/PortalSebrae/artigos/saiba-como-eliminardesperdicios-e-aumentar-a-produtividade-daempresa,ba2c4bc801ed6510VgnVCM1000004c00210aRCRD>. Acesso em: 23 ago. 2019.

[11] SILVA, M.; HAYASHI, C. M.; HAYASHI, M. P. I. da. Análise bibliométrica e cientométrica: desafios para especialistas que atuam no campo. InCID: Revista de Ciência da Informação e Documentação, v. 2, n. 1, p. 110-129, 2011

[12] SLACK, N.; CHAMBERS, S.; HARIAND, C.; HARRISON, A.; JOHNSTON, R. Administração da Produção. $3^{\text {a }}$ ed. São Paulo, Atlas, 2009.

[13] VIANA, J. J. Administração de materiais: um enfoque prático. 1. Ed. São Paulo: Atlas, 2006.

\section{APÊNDICE A}

ANDRIOLO, A; BATTINI, D.; PERSONA, A., \& SGARBOSSA, F. A new bi-objective approach for including ergonomic principles into EOQ model. International Journal of Production Research, v. 54, n.9, p. 2610-2627, 2016.

BATTINI, D.; GLOCK, C. H.; GROSSE, E. H., PERSONA, A., \& SGARBOSSA, F. Ergo-lotsizing: Considering ergonomics in lot-sizing decisions. IFAC-PapersOnLine, v. 48, n.3, p. 326-331, 2015. 
BONNEY, M. e JABER, M. Y. Environmentally responsible inventory models: Non-classical models for a non-classical era. International Journal of Production Economics, v. 133, n. 1, p. 4353, 2011.

BRAHIMI, N. e AOUAM, T. Multi-item production routing problem with backordering: a MILP approach. International Journal of Production Research, v. 54, n.4, p. 1076-1093, 2016.

CHUA, G. A.; MOKHLESI, R. e SAINATHAN, A. Optimal discounting and replenishment policies for perishable products. International Journal of Production Economics, v. 186, p. 8-20, 2017.

CLARK, A. J. \& SCARF, H. Optimal policies for a multi-echelon inventory problem. Management science, v.6, n. 4, p. 475-490, 1960.

CUI, L.; DENG, J.; LIU, F.; ZHANG, Y.; \& XU, M. Investigation of RFID investment in a single retailer two-supplier supply chain with random demand to decrease inventory inaccuracy. Journal of cleaner production, v. 142, p. 2028-2044, 2017.

FICHTINGER, J; RIES, J.M.; GROSSE, E.H.; \& BAKER, P. Assessing the environmental impact of integrated inventory and warehouse management. International Journal of Production Economics, v. 170, p. 717-729, 2015.

FULLERTON, R. R.; KENNEDY, F. A.; WIDENER, S. K. Management accounting and control practices in a lean manufacturing environment. Accounting, Organizations and Society, v. 38, n. 1, p. 50-71, 2013.

HAN, W \& WANG, J. The impact of cooperation mechanism on the chaotic behaviours in nonlinear supply chains. European Journal of Industrial Engineering, v. 9, n. 5, p. 595-612, 2015.

HOPP, W. J. e SPEARMAN, M. L. To pull or not to pull: what is the question? Manufacturing \& service operations management, v.6, n. 2, p. 133-148, 2004.

KULIŃSKA, E.; ODLANICKA-POCZOBUTT, M. e KULIŃSKA, K. Diagnosis of operational malfunctions and failures by the FMEA method in the industrial robots sector. Research in Logistics \& Production, v 8, 2018.

LI, Q.; YU, P. e WU, X. Shelf life extending packaging, inventory control and grocery retailing. Production and Operations Management, v. 26, n. 7, p. 1369-1382, 2017.

MAKKAR, S.; JHA, P. C. e ARORA, N. Single-source, single-destination coordination of EOQ Model for perishable products with quantity discounts incorporating partial/full truckload policy under fuzzy environment. In: International Journal of Production Economics. Springer, India, 2012. p. 971-982.76, 2008.

MAO, J. On-line inventory control in single-echelon systems with time-dependent environment over multiple periods. In: Decision and Control and European Control Conference, 2011. p. 76727677.

MOLINA, F.; MORABITO, R. e ARAÚJO, S. A. MIP models for production lot sizing problems with distribution costs and cargo arrangement. Journal of the Operational Research Society, v. 67, n. 11, p. 1395-1407, 2016.

OLÁH, J.; LAKNER, Z.; HOLLÓSI, D.; \& POPP, J. Inventory methods in order to minimize raw materials at the inventory level in the supply chain. European Journal of Industrial Engineering, v. 13, n. 4, 2017. 
PORRAS, E. e DEKKER, R. An inventory control system for spare parts at a refinery: An empirical comparison of different re-order point methods. European Journal of Operational Research, v. 184, n. 1, p. 101-132, 2008.

SARACOGLU, O.; ARSLAN, M. C. e TURKAY, M. Aggregate planning problem from sustainability perspective. In: Advanced Logistics and Transport (ICALT), 2015 4th International Conference on. IEEE, 2015. p. 181-186.

TÜRKAY, M. Environmentally conscious supply chain management. Transportation, v.1, n. 32, p, 2008

YAP, M. H. T. e CHEN, N. Understanding young Chinese wine consumers through innovation diffusion theory. Tourism and hospitality management, v. 23, n. 1, p. 51-68, 2017.

As Referências Bibliográficas devem seguir as normas da ABNT/NBR 6023. 